\title{
Regard complémentaire - Unité et diversité du didactique
}

Unity and diversity of didactics

\section{Christian Orange}

\section{(2) OpenEdition \\ Journals}

Édition électronique

URL : http://journals.openedition.org/educationdidactique/1897

DOI : 10.4000/educationdidactique.1897

ISSN : 2111-4838

Éditeur

Presses universitaires de Rennes

\section{Édition imprimée}

Date de publication : 30 juillet 2014

Pagination : 85-90

ISSN : 1956-3485

\section{Référence électronique}

Christian Orange, «Regard complémentaire - Unité et diversité du didactique », Éducation et didactique [En ligne], 8-1 | 2014, mis en ligne le 15 septembre 2016, consulté le 21 avril 2019. URL : http:// journals.openedition.org/educationdidactique/1897; DOI : 10.4000/educationdidactique.1897 


\section{UNITÉ ET DIVERSITÉ DU DIDACTIQUE}

Christian Orange

Université Libre de Bruxelles, CRSE ; CREN, SAVE, Université de Nantes 
Voilà un titre de didacticien de la biologie ${ }^{1}$. Ou plutôt d'un didacticien qui doit sa formation à la biologie puis à la didactique de la biologie. Peut-on échapper à son histoire? Les didactiques le peuventelles? Il s'agit pour moi de réagir aux présentations et discussions qui ont constitué la journée d'étude «Didactique et/ ou didactiques? D’une question polémique à la construction d'un espace de problématisation » proposée par l'ARCD et la revue Éducation et Didactique. La richesse des échanges et mes interrogations propres me conduisent nécessairement à ne prendre en compte qu'une petite partie de ce qui s'est dit.

Commençons tout d'abord par nous étonner de la question qui a organisé cette journée : didactique et/ ou didactiques. Étonnement non comme un reproche aux organisateurs - il s'agit bien d'une question en débat - mais étonnement qu'un tel débat existe. En effet, entend-on dans d'autres domaines scientifiques de telles discussions ? Il y a bien eu, me semble-t-il, au temps des «maths modernes », le débat mathématique vs mathématiques; n'est-ce pas, à l'époque, autour l'idée d'une possible construction axiomatique globale (cf. Bourbaki) que portait la controverse? Mais si on parle de biologie, de physique, de linguistique, on dit aussi bien sciences biologiques, sciences physiques, sciences du langage. Le pluriel ne gêne personne, au contraire. On demandera facilement à un biologiste de quelle sorte de biologie il s'occupe (biologie moléculaire, physiologie, diversité biologique, écologie, etc.) ; et à un historien s'il travaille sur l'antiquité ou s'il est médiéviste, moderne, ou contemporain; il y a donc des biologies et des histoires, ce qui n'empêche pas les chercheurs de se dire historien ou biologiste. Et surtout, tout cela ne fait pas débat.

D'un autre côté, le fait même que les différentes didactiques se nomment ainsi (didactique de...) ne pointe-il pas une unité acceptée et reconnue? Quant à la journée d'aujourd'hui, elle est la preuve que nous sommes un certain nombre à penser faire partie d'une même communauté, même si nous ne mettons pas tous didactique au singulier. D'où vient donc le problème ? De l'histoire sans doute, comme l'ont pointé plusieurs intervenants, chaque didactique ayant fait son chemin à partir des disciplines scolaires ou universitaires respectives. Ce qui, dans un premier temps, a rendu plus ou moins difficiles les échanges d'une didactique à l'autre et, dans un second temps, a fait penser à certains que ce cloi- sonnement était seul responsable du fait que toutes les didactiques n'adoptaient pas leur cadre théorique. Bref, nous étions entre ignorance et refus de prise en compte de la diversité. Il me semble que nous n'en sommes plus là, en partie grâce au travail au sein de l'ARCD. Mais la question demeure et les contributions de cette journée nous donnent des éléments pour en comprendre les raisons et repérer les problèmes qui s'y cachent.

Nous ne reprendrons pas directement les arguments développés sur le singulier et le pluriel. Cependant, il apparaît que cette question s'est développée de différentes façons dans les communications et les échanges. Deux thèmes retiendront particulièrement notre attention qui se sont retrouvés à plusieurs reprises. L'un ne semble pas concerner la question annoncée mais est fondamental : il s'agit de la délimitation du champ de la didactique ; l'autre correspond à la notion de didactique de discipline. Je vais discuter ces deux points à partir de ce qui s'en est dit et de quelques réflexions qui me sont alors venues. Cela permettra de conclure à une nécessaire unité des didactiques et à la nécessaire diversité du et de la didactique.

\section{LA DIDACTIQUE : PARLONS-NOUS TOUS DE LA MÊME CHOSE ?}

Tous les intervenants ne donnent pas la même définition de la didactique et/ ou du didactique et ne discutent donc pas de l'organisation du même champ d'investigation.

D’un point de vue général, Jean-Louis Martinand fait remarquer justement qu'il y a plusieurs orientations pour la didactique si on s'intéresse aux acteurs, à ceux qui se disent didacticiens : la didactique praticienne ou pragmatique (celle des enseignants), la didactique normative des inspecteurs, la didactique critique et prospective. Nous ne nous intéressons ici, compte tenu de la construction de cette journée autour d'une revue scientifique et d'une association de chercheurs, qu'à la didactique ou aux didactiques en tant de disciplines de recherche, ce qui correspond à la troisième catégorie de Jean-Louis Martinand ou à un sous-ensemble de cette catégorie ; on pourrait parler de la didactologie, nous en resterons à la didactique.

Cette première restriction faite, il reste des différences importantes dans la délimitation de la didac- 
tique scientifique. Bernard Schneuwly définit l'objet de la didactique comme " la transmission ou la diffusion de savoirs dans des institutions spécialisées à cette fin »(Schneuwly, dans ce numéro). Yves Chevallard étend le domaine du didactique à tous les faits didactiques qui, selon lui, regroupent l'ensemble des situations où une personne ou une institution intervient pour aider une personne ou une institution à accéder à certaines connaissances; y compris lorsque les deux personnes ou institutions ne sont qu'une. Ce qui conduit à l'affirmation que le didactique (objet de la didactique comme science) est partout dans la société. Cela s'admet volontiers car le didactique ainsi défini est au fondement même de toute culture. Le projet est ambitieux. Reste qu'une discipline de recherche se définit aussi bien par ce qu'elle n'est pas que par son objet. Qu'est-ce qui, dans ce cas, ne relèverait pas de la didactique dans les sciences humaines? Plus largement, tout travail scientifique ayant pour but d'aider telle instance, voire l'ensemble de l'humanité, à accéder à de nouveaux savoirs, relèverait du didactique; la didactique devient la sciences des sciences, englobant du même coup toutes les épistémologies : l'affaire n'est pas mince !

La position de Bernard Schneuwly, plus modeste, fait ressortir deux aspects qui sont anthropologiquement et sociologiquement importants, tout en permettant de décider ce qui ne relève pas du didactique et en précisant donc l'objet d'étude de la didactique. Il y a d'abord, et explicitement, l'existence d'institutions spécialisées dans la transmission des savoirs que l'on peut, dans un premier temps, nommer écoles, tout en ayant conscience de laisser ainsi de côté les institutions dédiées à l'éducation non formelle. Comme l'écrit J.-L. Martinand » quels que soient les champs disciplinaires, la didactique suppose un contexte de type scolaire " (Martinand, 1994, p. 13). Les écoles n'existent pas dans toute société ou pour tous les individus et leur existence semble fortement liée à l'usage et à la nécessité de l'écrit, ce qui donne une signification particulière à la textualité dans les faits didactiques (Rey, 2002). Le second point important est que le didactique est un objet non seulement anthropologique mais social : c'est une société qui décide la mise en place d'institutions dédiées à la diffusion des savoirs; et elle organise cette diffusion dans des écoles qui fonctionnent en communautés d'enseignement et d'apprentissage, avec les rôles dédiés de professeurs et d'élèves. Le regroupement des élèves dans des classes n'est pas qu'une disposition pratique ou pécuniaire car il s'agit bien qu'ils se construisent un vécu commun d'entrée dans la culture, ce qui rejoint une nécessité didactique et épistémologique : Qu'est-ce donc qu'apprendre par l'esprit ? C'est faire société » écrit Alain (1932, Propos XXVI). Et Bachelard (1938) insiste: " pour que la science objective soit pleinement éducatrice, il faudrait que son enseignement fût socialement actif » (p. 244).

Cette délimitation du domaine de recherche que propose Bernard Schneuwly est donc cohérente et pertinente épistémologiquement : elle identifie bien, selon moi, problème fondamental qui peut instituer un champ scientifique. Cela a donc un sens de parler de didactique scolaire, au risque du pléonasme, pour marquer la distinction avec une définition plus large proposée par certains. On trouvera dans l'intervention d'Yves Reuter d'autres arguments plaidant dans le sens des liens forts entre la didactique et l'école. Dans cette acception, à laquelle j'adhérerais volontiers, si le travail à la maison et les cours particuliers relèvent certainement du didactique, on ne peut pas en dire autant du préceptorat.

Une autre variation apparaît dans les différents positionnements des intervenants sur la ou les didactiques. Elle concerne la nature même des recherches didactiques. Les considère-t-on comme des recherches scientifiques ou comme des recherches technologiques. Jean-Louis Martinand, pointant sa convergence avec Bronckart, fait de la didactique une technologie culturelle et parle de discipline de génie ; on serait donc sur des études de conception et d'intervention. Yves Chevallard considère de toute évidence la didactique comme une science fondamentale. Sans être déterminants, ces positionnements ont une nécessaire influence sur la façon de penser les liens des recherches didactiques avec l'école et sur la délimitation du champ.

Ainsi la discussion sur le singulier ou le pluriel est l'occasion de croiser des points de vue différents sur ce qu'est la didactique, bien au-delà d'une simple affaire de dénomination. Il en ressort des questions qui sont au moins aussi indispensables à mettre au travail que celle de départ. On ne peut nier qu'une certaine diversité existe dont le lissage ne saurait se décréter car elle représente autant de points problématiques qui justifient à eux seuls le travail de la communauté des chercheurs en didactiques. Le « $\mathrm{s}$ » est ici pour marquer qu'aujourd'hui la didactique rassemble nécessairement des didactiques. 
«Les didactiques sont fondamentalement plurielles» note Jean-Louis Martinand (dans ce numéro). C'est d'autant plus vrai qu'il faut intégrer à la discussion la place à donner à la didactique (ou aux didactiques) des éducations non formelles et à la (aux ?) didactique(s) professionnelle(s). Par exemple, Pastré, Mayen et Vergnaud (2006) écrivent : « La didactique professionnelle a pour but d'analyser le travail en vue de la formation des compétences professionnelles » (p. 145). Comment une telle définition s'inscrit-elle dans la discussion précédente ? Implique-t-elle, par exemple, l'existence d'institutions spécialisées dans la formation professionnelle? Peut-on penser que le didactique est absent des situations de travail, question que pose Laurent Veillard ${ }^{2}$, depuis l'étude des dynamiques de formation professionnelle?

Est-il alors pertinent de vouloir dès aujourd'hui détenir une théorie unifiée du didactique ? La construction d'une telle théorie est un pari certainement heuristique; c'est un programme de recherche passionnant et vraisemblablement nécessaire, comme le dit Helmut Johannes Vollmer. Mais une théorie ne peut pas s'imposer a priori. Car il ne s'agit pas simplement de trouver un moyen général de décrire les faits didactiques à partir d'une approche déductive : il faut encore que cette théorie, confrontée sans relâche à des cas, permette de construire ou de reconstruire l'ensemble des problèmes didactiques et conduise ainsi à une intelligence qui subsume celles que procurent les différents cadres didactiques. Pour cela, elle doit non seulement conduire à la définition de problèmes nouveaux mais également rendre compte de la diversité des épistémologies mobilisées en didactiques ou la réduire de façon convaincante. Vaste programme...

\section{DIDACTIQUE(S) DES DISCIPLINES... OU PAS}

En lien avec la discussion précédente, une autre se développe qui, d'une certaine façon, s'y inscrit. Alors que le champ de recherche didactique s'est constitué à partir des didactiques des disciplines, est-il encore pertinent de parler de ou depuis les didactiques des disciplines?

Yves Reuter se prononce pour le pluriel des didactiques et pour un lien fort avec l'organisation en disciplines ; ses raisons sont épistémologiques (focalisation sur les contenus), historiques et institutionnelles. Selon lui, didactique disciplinaire est pléonastique.
Au contraire, Yves Chevallard voit dans cet attachement de la didactique aux disciplines scolaires une trace de l'histoire du domaine et le considère comme une maladie infantile. Il s'étonne de cette référence acritique à des objets de la société, les disciplines.

Pour Jean-Louis Martinand, les didactiques sont fondamentalement plurielles et, tout au moins pour l'enseignement secondaire, en lien avec les disciplines. Il parle ainsi, depuis de nombreuses années, de didactiques de discipline. "Didactique et discipline sont plus que des mots accolés en didactique des disciplines : ce sont des concepts interdépendants. Du point de vue de la recherche, les didactiques ne se développent que sur des disciplines qu'elles ne peuvent transformer mais qu'elles acceptent comme une donnée " (Martinand, 1994, p. 12). Aujourd'hui comme alors, il met en avant la responsabilité des didactiques par rapport aux contenus mais semble avoir un peu infléchi sa position en ajoutant : "que ces contenus soient disciplinaires ou non " (Martinand, dans ce numéro).

Trois positions donc, contrastées, qu'il faudrait compléter, notamment par le point de vue que présente Helmut Johannes Vollmer. En Allemagne, les didactiques correspondent aux matières enseignées mais il plaide pour l'émergence d'une « didactique disciplinaire généralisée ». Une remarque s'impose d'emblée: les positions sur les liens entre didactique et discipline dépendent pour une part du contexte. L'organisation universitaire varie d'un pays à l'autre. Dans certains cas (Belgique, Suisse, par exemple), les didacticiens sont installés principalement dans les facultés disciplinaires, en lien avec la formation des enseignants du secondaire, ce qui les conduit à être des didacticiens de discipline; quant à ceux qui, dans ces pays, sont, pour une raison ou une autre, au sein des sciences de l'éducation, ils doivent adopter un cadre plus large que celui d'une didactique disciplinaire.

Plus fondamentalement, il me semble que l'on peut développer la discussion autour de la fonction essentielle d'une discipline telle que la rappelle Yves Reuter, reprenant les propos de Chervel (1988) : produire de l'enseignable. Si on va dans cette direction, il est clair que les disciplines enseignées aujourd'hui ont une part de contingence; la preuve en est qu'elles varient d'un pays à l'autre. Cependant une discipline, considérée comme un système à produire de l'enseignable, est nécessairement adaptée à l'école où elle 
trouve place, tout comme elle agit sur cette école. Elle répond donc à un certain nombre de nécessités fonctionnelles que l'on peut étudier à partir du processus de transposition didactique (que la référence soit un texte de savoir ou des pratiques), des orientations épistémologiques qui la caractérise, du concept de forme scolaire, etc. Tout ne peut pas faire discipline et une discipline, de par les nécessités fonctionnelles qui la contraignent, est un objet social, historique et épistémologique qui a une logique propre.

Alors s'il est juste, comme le fait Yves Chevallard, d'être critique sur l'attachement de didacticiens à une discipline établie, on ne peut nier l'intérêt d'étudier les conditions d'existence d'une discipline, scolaire ou universitaire ${ }^{3}$, en tant que système à produire de l'enseignable, donc d'étudier les conditions d'un apprentissage et d'un enseignement dans cette discipline. A condition cependant de ne pas s'en tenir aux disciplines présentes ici et maintenant, comme si elles étaient des entités figées, mais en incluant l'étude les disciplines passées et surtout possibles: une science ne se construit pas sur des constats mais par l'exploration des possibles.

Du coup, le concept de didactique d'une discipline a un sens, construit par ce problème » d'enseignabilité « qui peut être travaillé aussi bien fondamentalement qu'avec des perspectives d'intervention. Cela dit, si toutes les disciplines actuelles, passées et à venir répondent à des nécessités fonctionnelles communes, elles ont des contraintes spécifiques, dues notamment à la particularité de ce qu'elles produisent comme enseignable. Elles ne fonctionnent donc pas toutes semblablement et n'évoluent pas de la même façon : les disciplines sont nécessairement divergentes et plurielles car c'est leur raison d'être. Ce qui n'empêche pas de rechercher et de trouver des convergences fonctionnelles. Voilà le beau programme de recherche de la didactique comparée.

En croisant ainsi différents points de vue exprimés lors de la journée et faisant des choix que nous avons essayé d'expliquer, nous pourrions dire que les didactiques étudient les systèmes (les disciplines) - systèmes dynamiques, avec histoire et mémoire - produisant de l'enseignable dans des institutions dédiées. Elles portent en elles la nécessité, apparue depuis le XIX ${ }^{\mathrm{e}}$ siècle, de renoncer à une organisation des enseignements à une seule dimension (les humanités), donc de passer à des programmations en parallèle. Cela conduit à s'interroger sur cette nécessité de différencier des disciplines pour produire de l'enseignable, en allant au-delà du slogan simpliste de l'interdisciplinarité ; mais aussi à questionner ce qui est enseignable et ce que veut dire produire de l'enseignable, donc à travailler les conditions de possibilité de l'enseignable dans tel domaine : on peut le faire empiriquement, à partir des disciplines actuellement en vigueur, par exemple en étudiant les pratiques ordinaires ; on peut également construire et étudier du neuf, plus ou moins éloigné des disciplines telles qu'elles sont, en développant des phénoménotechniques (Bachelard, 1949 ; Brousseau, 1981). A chaque fois, cela ne peut se faire sans analyse épistémologique, sauf à penser que » l'enseignabilité« ne dépend que de l'habilité du professeur et nullement des savoirs en jeu, ce qui ne peut être une position didactique.

Cette façon de voir la ou les didactiques permet d'avancer sur certaines questions et d'en repérer d'autres. Ainsi, le fait que l'école primaire ne fonctionne pas sous le régime des disciplines du secondaire n'empêche pas une approche didactique, car là aussi nous avons affaire à des systèmes (domaines ou autres) qui produisent de l'enseignable et qui fonctionnent, au moins en partie, en parallèle. Quant aux » éducations à », qui ne prennent pas la forme de disciplines, on peut s'interroger sur leur capacité à produire de l'enseignable. Autre question, plus générale : à quelles conditions peut-on dire que de l'enseignable a été produit?

Sur ce dernier point, plusieurs intervenants ont mobilisé la notion de contenu disciplinaire. Yves Reuter y met, dans leur diversité, savoirs, savoirfaire, rapports à, valeurs, manières de penser, de discourir, d'agir. Il n'est pas sûr que tous les didacticiens qui utilisent ce terme y mettent la même chose. Comme l'écrit Bernard Rey (2007), » il y a des mots qui servent à combler des trous ou qui désignent un quelque chose dont il n'est pas opportun ni possible de préciser la spécificité. [...] Le mot contenu est de ceuxlà. » (p. 130). Plus largement on voit la difficulté de définir ce qui s'enseigne. C'est notamment la question du rapport entre texte et pratiques à l'école (Rey, 2007) qui se pose, comme l'a par ailleurs souligné Laurent Veillard. Peut-il être le même dans toutes les disciplines et dans tous les apprentissages, qu'ils soient "généraux » ou professionnels? Question que les concepts de transposition et de pratique sociale de référence éclairent mais ne règlent pas à eux seuls, selon moi. Il faut leur ajouter notamment une épistémologie des savoirs scolaires (ou professionnels) 
consistante, qui prenne en compte les finalités de leur apprentissage, et la mettre sans cesse au travail sur des cas. "Au fond, dit Bernard Schneuwly, les didacticiens analysent avant tout le processus continu et inéluctable de transformation du savoir » (Schneuwly, dans ce numéro).

La levée des ambiguités sur ce qui s'enseigne, sur ce qui peut s'enseigner et sur ce qui peut s'apprendre est bien au cœur de la recherche didactique. C'est une question particulièrement importante au moment où l'invasion des programmes par l'approche par compétences augmente encore, si c'était possible, la confusion entre ce qui se fait en classe, ce qui s'y enseigne et ce qui s'y apprend.

\section{CONCLUSION}

Les différentes interventions de la journée ont permis de mettre en avant des problèmes didactiques fondamentaux, même s'ils n'ont pas pu tous être thématisés et développés à cette occasion. En cela la question de départ (didactique ou didactiques) a été efficace. En tout cas la diversité des approches didactiques est apparue, qui justifie le pluriel, tout comme est apparue la possibilité de discussions de fond entre didacticiens, ce qui plaide en faveur de l'existence d'une didactique : nous ne sommes pas sur des paradigmes incommensurables et toutes les didactiques ont un intérêt scientifique à vivre et à se développer au sein de la didactique. En acceptant de travailler ensemble, les chercheurs des différentes didactiques se donnent les moyens d'échapper aux deux risques contre lesquels Bachelard nous met en garde « L'esprit scientifique peut se fourvoyer en suivant deux tendances contraires : l'attrait du singulier et l'attrait de l'universel » (Bachelard, 1938, p. 60).

Quand je parle ici des didactiques (au pluriel), je ne pense pas uniquement à la diversité que représentent les didactiques de discipline. Même si, de mon point de vue, les disciplines - non pas dans leur existence empirique mais dans leur existence théorique, dans leur fonction - restent au cœur des didactiques, il n'est pas sûr qu'elles constituent une diversité plus grande et plus riche que d'autres différences, comme celle, par exemple, entre ceux qui travaillent sur les pratiques ordinaires et ceux qui construisent et étudient de nouveaux phénomènes didactiques.

Le didactique est donc divers mais présente une unité anthropologique et sociologique. Les didac- tiques ont des origines et des histoires différentes, différence renforcée par la diversité du didactique, mais ont suffisamment de points communs pour échanger scientifiquement. Nous devons nous intéresser à cette diversité : c'est notre richesse commune.

\section{NOTES}

1. "L'unité et la diversité du vivant «est un intitulé des programmes de sciences expérimentales et technologies du CE2, CM1, CM2 français de 2008. Plus largement, unité et diversité est une problématique constitutive de la biologie. 2. La journée d'étude du 10 juin 2013 comportait également une intervention de Laurent Veillard (ICAR, Université de Lyon 2), intitulée "Intérêt d'une analyse didactique comparatiste en formation professionnelle initiale », qui n'a pas pu faire l'objet d'un texte dans ce numéro.

3. Les disciplines scolaires et universitaires ne sont pas nécessairement les mêmes, malgré les contraintes sociales de justification et celles portant sur formation universitaire des enseignants.

\section{RÉFÉRENCES}

Alain (1932). Propos sur l'éducation. Paris : PUF

Bachelard, G. (1938). La formation de l'esprit scientifique. Paris : Vrin.

Bachelard, G. (1949). Le rationalisme appliqué. Paris : PUF Brousseau, G. (1981). Problèmes de didactique des décimaux. Recherche en Didactique des Mathématiques, 2, 3 , 37-127.

Chervel, A. (1988). Lhistoire des disciplines scolaires. Réflexions sur un domaine de recherche. Histoire de l'éducation, 38, 59 -119.

Martinand, J.-L. (1994). Didactique des sciences et formation des enseignants, notes d'actualité. Les sciences de l'éducation, pour l'ère nouvelle. 1994, 1, 16-24.

Pastré, P., Mayen, P. \& Vergnaud, G. (2006). La didactique professionnelle, note de synthèse. Revue Française de Pédagogie, 154, 145-198.

Rey, B. (2002). Diffusion des savoirs et textalité. Recherche et formation, 40, 43-57.

Rey, B. (2007). Autour du mot « contenu». Recherche et formation, 55, 119-133. 